\title{
The number of vertices of a Fano polytope ${ }^{1}$
}

\author{
Cinzia Casagrande
}

Let $X$ be a normal, complex, projective variety of dimension $n$. Assume that $X$ is Gorenstein and Fano, namely the anticanonical divisor $-K_{X}$ of $X$ is Cartier and ample. The pseudo-index of $X$ was introduced in [18] as

$$
\iota_{X}:=\min \left\{-K_{X} \cdot C \mid C \text { rational curve in } X\right\} \in \mathbb{Z}_{>0} .
$$

By Mori theory we know that $\iota_{X} \leq n+1$ when $X$ is smooth, and $\iota_{X} \leq 2 n$ in general (see for instance [8, Theorems 3.4 and 3.6]).

The object of this paper is to give some bounds on the Picard number $\rho_{X}$ of $X$, in terms of $n$ and $\iota_{X}$, when $X$ is toric and $\mathbb{Q}$-factorial ${ }^{2}$. More precisely, we will prove the following:

Theorem 1. Let $X$ be a $\mathbb{Q}$-factorial, Gorenstein, toric Fano variety of dimension $n$, Picard number $\rho_{X}$ and pseudo-index $\iota_{X}$. Then:

(i) $\rho_{X} \leq 2 n$, with equality if and only if $n$ is even and $X \cong\left(S_{3}\right)^{\frac{n}{2}}$, where $S_{3}$ is the blow-up of $\mathbb{P}^{2}$ at three non collinear points;

(ii) $\rho_{X}\left(\iota_{X}-1\right) \leq n$, with equality if and only if $X \cong\left(\mathbb{P}^{\iota_{X}-1}\right)^{\rho_{X}}$.

Part (ii) of Theorem [1 has been conjectured in [5] for any smooth Fano variety $X$, generalizing a conjecture by $\mathrm{S}$. Mukai. For such $X$, (iii) is known in the cases $\iota_{X} \geq \frac{1}{2} n+1$ [18, 7], 14], $n \leq 4$ [5], $n=5$ [1], and, provided that $X$ admits an unsplit covering family of rational curves, $\iota_{X} \geq \frac{1}{3} n+1$ [1]. For $X$ smooth and toric, (iii) was already known in the cases $n \leq 7$ or $\iota_{X} \geq \frac{1}{3} n+1$ [5].

For a smooth toric Fano $X$, (iil) was conjectured by V. V. Batyrev (see [10. page 337]) and was already known to hold up to dimension 5 (for $n \leq 4$ thanks to the classifications [2, 17, 4, 15], and for $n=5$ it is [6. Theorem 4.2]). Recently B. Nill [13] has extended this conjecture to the $\mathbb{Q}$-factorial Gorenstein case, and has shown (ii) for a certain class of $\mathbb{Q}$-factorial, Gorenstein toric Fano varieties (see on page 3i).

Observe that the bound in (iil) does not hold for non toric Fano varieties, already in dimension two. It is remarkable that in the non toric case, there is no known bound for the Picard number of a smooth Fano variety in terms of its dimension (at least to our knowledge). If $S$ is a surface obtained by blowing-up

\footnotetext{
${ }^{1} 2000$ Mathematics Subject Classification: 52B20, 14M25, 14J45

${ }^{2}$ For every Weil divisor $D$ there exists $m \in \mathbb{Z}_{>0}$ such that $m D$ is Cartier.
} 
$\mathbb{P}^{2}$ at eight general points, for any even $n$ the variety $S^{\frac{n}{2}}$ has Picard number $\frac{9}{2} n$, and one could conjecture this is the maximum for any dimension $n$ (see [9, page 122]).

For $X$ smooth, toric, and Fano, V. E. Voskresenskiu and A. Klyachko have shown that $\rho_{X} \leq n^{2}-n+1$ [16, Theorem 1]; O. Debarre has improved this bound in $\rho_{X} \leq 2+\sqrt{(2 n-1)\left(n^{2}-1\right)}$ 9, Theorem 8].

The common approach to these questions in the toric case, is to associate a so-called "reflexive" polytope to a Gorenstein toric Fano variety, and to combine the techniques coming from geometry with the ones coming from the theory of polytopes.

We recall some basic notions on reflexive polytopes and toric Fano varieties; we refer the reader to [10], 9] and references therein for more details. Let $N \cong \mathbb{Z}^{n}$ be a lattice and let $M:=\operatorname{Hom}_{\mathbb{Z}}(N, \mathbb{Z})$ be the dual lattice. Set $N_{\mathbb{Q}}:=N \otimes_{\mathbb{Z}} \mathbb{Q}$ and $M_{\mathbb{Q}}:=M \otimes_{\mathbb{Z}} \mathbb{Q} ;$ for $x \in N_{\mathbb{Q}}$ and $y \in M_{\mathbb{Q}}$ we denote by $\langle x, y\rangle$ the standard pairing. For any set of points $x_{1}, \ldots, x_{r} \in N_{\mathbb{Q}}$, we denote by $\operatorname{Conv}\left(x_{1}, \ldots, x_{r}\right) \subset N_{\mathbb{Q}}$ their convex hull.

Let $P \subset N_{\mathbb{Q}}$ be a lattice polytope of dimension $n$ containing the origin in its interior. We denote by $V(P)$ the set of vertices of $P$. The dual polytope of $P$ is defined as

$$
P^{*}:=\left\{y \in M_{\mathbb{Q}} \mid\langle x, y\rangle \geq-1 \text { for all } x \in P\right\} .
$$

$P$ is called a reflexive polytope if $P^{*}$ is a lattice polytope; if so, also $P^{*}$ is reflexive and $\left(P^{*}\right)^{*}=P$. Reflexive polytopes were introduced in [3]; their isomorphism classes are in bijection with isomorphism classes of Gorenstein toric Fano varieties.

Let $P$ be a reflexive polytope of dimension $n$; we denote by $X_{P}$ the associated $n$-dimensional Gorenstein toric Fano variety. The fan of $X_{P}$ is given by the cones over the faces of $P$ in $N_{\mathbb{Q}}$.

Many geometric properties of $X_{P}$ can be read from $P$. In particular, $X_{P}$ is $\mathbb{Q}$-factorial if and only if $P$ is simplicial ${ }^{3}$, while $X_{P}$ is smooth if and only if the vertices of every facet of $P$ are a basis of the lattice. In this last case, we say that $P$ is smooth.

Example. In dimension two, let $e_{1}, e_{2}$ be a basis of $N$, and $e_{1}^{*}, e_{2}^{*}$ the dual basis of $M$. Consider $P:=\operatorname{Conv}\left(e_{1}, e_{2},-e_{1}-e_{2}\right)$; its dual polytope is $P^{*}=\operatorname{Conv}\left(2 e_{1}^{*}-\right.$ $\left.e_{2}^{*}, 2 e_{2}^{*}-e_{1}^{*},-e_{1}^{*}-e_{2}^{*}\right)$.

\footnotetext{
${ }^{3} \mathrm{~A}$ polytope is simplicial if the vertices of every facet are linearly independent, where a facet is a proper face of maximal dimension.
} 

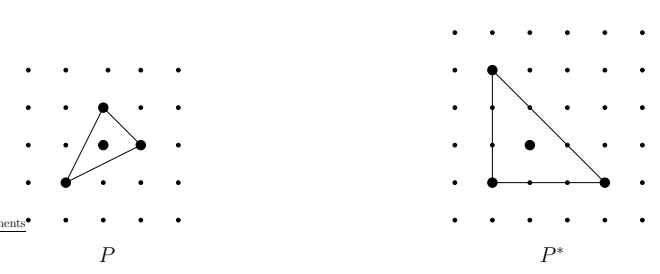

Both polytopes are reflexive, the associated surfaces are $X_{P}=\mathbb{P}^{2}$ and $X_{P^{*}}=$ $\left\{x y z=w^{3}\right\} \subset \mathbb{P}^{3}$. The surface $X_{P^{*}}$ has three singular points of type $A_{2}$. We have $\iota_{X_{P}}=3$, while $\iota_{X_{P^{*}}}=1$.

We denote by $|A|$ the cardinality of a finite set $A$. When $P$ is simplicial, the number of vertices $|V(P)|$ of $P$ is equal to $\rho_{X_{P}}+n$.

Recall that there is a bijection between the vertices of $P^{*}$ and the facets of $P$; if $u \in V\left(P^{*}\right)$, we denote by $F_{u}$ the corresponding facet of $P$, namely $F_{u}:=\{x \in P \mid\langle x, u\rangle=-1\}$. We define

$$
\delta_{P}:=\min \left\{\langle v, u\rangle \mid v \in V(P), u \in V\left(P^{*}\right), v \notin F_{u}\right\} \in \mathbb{Z}_{\geq 0} .
$$

The pseudoindex $\iota_{X_{P}}$ is related to $\delta_{P}$ as follows.

Lemma 2. Let $P$ be a simplicial reflexive polytope and $X_{P}$ the associated Fano variety. Then $\iota_{X_{P}} \leq \delta_{P}+1$. If moreover $P$ is smooth, then $\iota_{X_{P}}=\delta_{P}+1$.

Theorem 1 is then a consequence of Lemma 2 and of the following:

Theorem 3. Let $P$ be a simplicial reflexive polytope of dimension $n$. Then:

(i) $|V(P)| \leq 3 n$, with equality if and only if $n$ is even and $X_{P} \cong\left(S_{3}\right)^{\frac{n}{2}}$;

(ii) if $\delta_{P}>0$, then $|V(P)| \leq n+\frac{n}{\delta_{P}}$, with equality if and only if the following two conditions hold:

a. $P=\operatorname{Conv}\left(Q_{1}, \ldots, Q_{r}\right)$ with $r=\frac{n}{\delta_{P}}$ and each $Q_{j} \subset N_{\mathbb{Q}}$ a reflexive lattice simplex of dimension $\delta_{P}$, the sum of whose vertices is zero;

b. if $H_{j}$ is the linear span of $Q_{j}$ in $N_{\mathbb{Q}}$, we have $N_{\mathbb{Q}}=H_{1} \oplus \cdots \oplus H_{r}$.

In [13, Theorem [3 (ii) is proven for a simplicial reflexive polytope $P$ for which there exists a vertex $u$ of $P^{*}$ such that $-u \in P^{*}$ [13, Theorem 5.8]. We refer the reader to [13] for a discussion on the number of vertices of a reflexive polytope in the non simplicial case.

Example. The polytopes $P$ and $P^{*}$ of the previous example have $\delta_{P}=\delta_{P^{*}}=2$, and both satisfy equality in (iii). Notice that $\iota_{X_{P^{*}}}<\delta_{P^{*}}+1$. 
We will first prove Theorem 3, then Lemma 2 and Theorem 1, For the proof, we will use the same technique as [16] and [9], and also some results from [13].

First of all, we need a property of pairs of vertices $v \in V(P)$ and $u \in V\left(P^{*}\right)$ with $\langle v, u\rangle=\delta_{P}$.

Let $P$ be a simplicial polytope of dimension $n$. We say that a vertex $v$ is adjacent to a facet $F=\operatorname{Conv}\left(e_{1}, \ldots, e_{n}\right)$ if $\operatorname{Conv}\left(v, e_{1} \ldots, \check{e}_{i}, \ldots, e_{n}\right)$ is a facet of $P$ for some $i=1, \ldots, n$.

Lemma 4. Let $P$ be a simplicial reflexive polytope and $v \in V(P), u \in V\left(P^{*}\right)$ such that $\langle v, u\rangle=\delta_{P}$. Then $v$ is adjacent to $F_{u}$.

Proof. This property is shown in [9, Remark 5(2)] and [13, Lemma 5.5] in the case $\delta_{P}=0$. The same proof works for the general case.

Proof of Theorem 3. First of all, observe that for any $u \in V\left(P^{*}\right)$ we have

$$
|\{v \in V(P) \mid\langle v, u\rangle=-1\}|=n \quad \text { and } \quad|\{v \in V(P) \mid\langle v, u\rangle=0\}| \leq n .
$$

In fact, since $P$ is simplicial, the facet $F_{u}$ contains $n$ vertices. Moreover, if $\langle v, u\rangle=0$, then $\delta_{P}=0$, and by Lemma 4 we know that $v$ is adjacent to $F_{u}$. Again, since $P$ is simplicial, $F_{u}$ has at most $n$ adjacent vertices, and we get (11).

The origin lies in the interior of $P^{*}$, so we can write a relation

$$
m_{1} u_{1}+\cdots+m_{h} u_{h}=0
$$

where $h>0, u_{1}, \ldots, u_{h}$ are vertices of $P^{*}$, and $m_{1}, \ldots, m_{h}$ are positive integers. Set $I:=\{1, \ldots, h\}$ and $M:=\sum_{i \in I} m_{i}$. For any vertex $v$ of $P$ define

$$
A(v):=\left\{i \in I \mid\left\langle v, u_{i}\right\rangle=-1\right\} \quad \text { and } \quad B(v):=\left\{i \in I \mid\left\langle v, u_{i}\right\rangle=0\right\} .
$$

Then observe that $\left\langle v, u_{i}\right\rangle \geq 1$ for any $i \notin A(v) \cup B(v)$. So for every $v \in V(P)$ we have

$$
\begin{aligned}
0 & =\sum_{i \in I} m_{i}\left\langle v, u_{i}\right\rangle=-\sum_{i \in A(v)} m_{i}+\sum_{i \notin A(v) \cup B(v)} m_{i}\left\langle v, u_{i}\right\rangle \\
& \geq-\sum_{i \in A(v)} m_{i}+\sum_{i \notin A(v) \cup B(v)} m_{i}=M-2 \sum_{i \in A(v)} m_{i}-\sum_{i \in B(v)} m_{i} .
\end{aligned}
$$

Summing over all vertices of $P$ we get

$$
\begin{aligned}
& M|V(P)| \leq 2 \sum_{v \in V(P)} \sum_{i \in A(v)} m_{i}+\sum_{v \in V(P)} \sum_{i \in B(v)} m_{i} \\
& =2 \sum_{i \in I} m_{i}\left|\left\{v \in V(P) \mid\left\langle v, u_{i}\right\rangle=-1\right\}\right|+\sum_{i \in I} m_{i}\left|\left\{v \in V(P) \mid\left\langle v, u_{i}\right\rangle=0\right\}\right|
\end{aligned}
$$


and using (11) this gives $|V(P)| \leq 3 n$.

Assume that $|V(P)|=3 n$. Then all inequalities above are equalities; in particular, for any $v$ and $u_{i}$ such that $\left\langle v, u_{i}\right\rangle>0$, we must have $\left\langle v, u_{i}\right\rangle=1$. Observe now that we can choose a relation as (2) involving all vertices of $P^{*}$, namely with $h=\left|V\left(P^{*}\right)\right|$ (see Remark [5), so $\langle v, u\rangle \in\{-1,0,1\}$ for every $v \in$ $V(P)$ and $u \in V\left(P^{*}\right)$. Then $P$ and $P^{*}$ are centrally symmetric.

Smooth centrally symmetric reflexive polytopes are classified in [16. Theorem $6]$, and the only case with $3 n$ vertices is for $n$ even and $X_{P} \cong\left(S_{3}\right)^{\frac{n}{2}}$. For the general case, we apply [13, Theorem 5.8].

Now assume that $\delta_{P}>0$ and let's prove (iii). For every vertex $v$ of $P$ we have $\left\langle v, u_{i}\right\rangle \geq \delta_{P}$ if $i \notin A(v)$; similarly to what precedes, we get

$$
\begin{aligned}
0 & =\sum_{i \in I} m_{i}\left\langle v, u_{i}\right\rangle=-\sum_{i \in A(v)} m_{i}+\sum_{i \notin A(v)} m_{i}\left\langle v, u_{i}\right\rangle \\
& \geq-\sum_{i \in A(v)} m_{i}+\delta_{P} \sum_{i \notin A(v)} m_{i}=\left(-\delta_{P}-1\right) \sum_{i \in A(v)} m_{i}+\delta_{P} M,
\end{aligned}
$$

namely $\frac{\delta_{P}}{\delta_{P}+1} M \leq \sum_{i \in A(v)} m_{i}$. This gives

$$
|V(P)| \frac{\delta_{P}}{\delta_{P}+1} M \leq \sum_{v \in V(P)} \sum_{i \in A(v)} m_{i}=n M \quad \text { and } \quad|V(P)| \leq \frac{n\left(\delta_{P}+1\right)}{\delta_{P}}=n+\frac{n}{\delta_{P}}
$$

Suppose that $|V(P)|=n+\frac{n}{\delta_{P}}$. Again, we can choose a relation as (2) involving all vertices of $P^{*}$. Then, since we must have all equalities in (13), we conclude that $\langle v, u\rangle \in\left\{-1, \delta_{P}\right\}$ for any $v \in V(P)$ and $u \in V\left(P^{*}\right)$.

Set $r:=\frac{n}{\delta_{P}}$. Fix $u \in V\left(P^{*}\right)$ and call $e_{1}, \ldots, e_{n}$ the vertices of $F_{u}$, and $f_{1}, \ldots, f_{r}$ the remaining vertices. Set $K:=\{1, \ldots, n\}$ and $J:=\{1, \ldots, r\}$. For any $k \in K$, the face $\operatorname{Conv}\left(e_{1}, \ldots, \check{e}_{k}, \ldots, e_{n}\right)$ lies on exactly two facets, one of which is $F_{u}$. Hence there exists a unique $\varphi(k) \in J$ such that

$$
F_{k}:=\operatorname{Conv}\left(f_{\varphi(k)}, e_{1}, \ldots, \check{e}_{k}, \ldots, e_{n}\right)
$$

is a facet of $P$. This defines a function $\varphi: K \rightarrow J$.

Since $P$ is simplicial, $e_{1}, \ldots, e_{n}$ is a basis of $N_{\mathbb{Q}}$; if $e_{1}^{*}, \ldots, e_{n}^{*}$ is the dual basis in $M_{\mathbb{Q}}$, we have $u=-e_{1}^{*}-\cdots-e_{n}^{*}$. Fix $k \in K$ and let $u_{k}$ be the vertex of $P^{*}$ such that $F_{k}=F_{u_{k}}$. We have

$$
\left\langle e_{i}, u_{k}\right\rangle=-1 \text { for all } i \in K \backslash\{k\} \text {, and }\left\langle e_{k}, u_{k}\right\rangle=\delta_{P},
$$

so $u_{k}=u+\left(\delta_{P}+1\right) e_{k}^{*}$. 
Now for any $j \in J$ we have

$$
\left\langle f_{j}, e_{k}^{*}\right\rangle=\frac{1}{\delta_{P}+1}\left(\left\langle f_{j}, u_{k}\right\rangle-\delta_{P}\right)= \begin{cases}-1 & \text { if } \varphi(k)=j \\ 0 & \text { otherwise. }\end{cases}
$$

This means $f_{j}+\sum_{k \in \varphi^{-1}(j)} e_{k}=0$. Finally, we have $\delta_{P}=\left\langle f_{j}, u\right\rangle=\left|\varphi^{-1}(j)\right|$, so as $j$ varies in $J$, the $\varphi^{-1}(j)^{\prime}$ 's give a partition of $K$ in $r$ subsets of cardinality $\delta_{P}$. Setting $Q_{j}:=\operatorname{Conv}\left\{f_{j}, e_{k} \mid k \in \varphi^{-1}(j)\right\}$, we see that $Q_{1}, \ldots, Q_{r}$ satisfy the properties claimed in (iii).

Remark 5 . Consider any polytope $Q \subset M_{\mathbb{Q}}$, of dimension $n$, containing the origin in its interior. Let $u$ be a vertex of $Q$ and let $F$ be the minimal face of $Q$ such that $-u$ is contained in the cone over $F$ in $M_{\mathbb{Q}}$. Then writing $-u$ as a linear combination of the vertices of $F$, we get a relation as (2) containing $u$. Summing enough relations of this type, one easily finds a relation $\sum_{u \in V(Q)} m_{u} u=0$ with all $m_{u}$ 's positive integers.

It is interesting to observe that when $Q$ is a reflexive polytope, there is a special relation:

$$
\sum_{u \in V(Q)} \operatorname{Vol}\left(F_{u}\right) u=0
$$

where $F_{u}$ is the facet of $Q^{*}$ corresponding to $u$, and $\operatorname{Vol}\left(F_{u}\right)$ is the lattice volume of $F_{u}$. This follows from a theorem by Minkowski, see [11, page 332] and [12, Lemma 4.9].

If $P$ is a smooth reflexive polytope, then all facets of $P$ are standard simplices, so (44) yields that the sum of all vertices of $P^{*}$ is zero. This remarkable fact can also be proven using the recent results on the factorization of birational maps between smooth toric varieties, and it was used for the proof of Theorem 3 in a previous version of this work.

Let $P$ be a simplicial reflexive polytope. We denote by $\mathcal{N}_{1}\left(X_{P}\right)$ the $\mathbb{Q}$-vector space of 1-cycles in $X_{P}$, with rational coefficients, modulo numerical equivalence. It is a well known fact in toric geometry (see for instance [19]) that there is an exact sequence

$$
0 \longrightarrow \mathcal{N}_{1}\left(X_{P}\right) \longrightarrow \mathbb{Q}^{V(P)} \longrightarrow N_{\mathbb{Q}} \longrightarrow 0
$$

so that $\mathcal{N}_{1}\left(X_{P}\right)$ is canonically identified with the group of linear rational relations among the vertices of $P$.

Moreover, if a class $\gamma \in \mathcal{N}_{1}\left(X_{P}\right)$ corresponds to a relation

$$
\sum_{v \in V(P)} m_{v} v=0, \quad m_{v} \in \mathbb{Q}
$$

then the anticanonical degree of $\gamma$ is $-K_{X_{P}} \cdot \gamma=\sum_{v \in V(P)} m_{v}$. 
Proof of Lemma 2. To show that $\iota_{X_{P}} \leq \delta_{P}+1$, we exhibit a rational curve in $X_{P}$ whose anticanonical degree is less or equal than $\delta_{P}+1$. Fix $v \in V(P)$ and $u \in V\left(P^{*}\right)$ such that $\langle v, u\rangle=\delta_{P}$. By Lemma $4 v$ is adjacent to $F_{u}$. Let $e_{1}, \ldots, e_{n}$ be the vertices of $F_{u}$; up to reordering, we can assume that $\operatorname{Conv}\left(v, e_{2}, \ldots, e_{n}\right)$ is a facet of $P$. Since $P$ is simplicial, $e_{1}, \ldots, e_{n}$ is a basis of $N_{\mathbb{Q}}$; if $e_{1}^{*}, \ldots, e_{n}^{*}$ is the dual basis of $M_{\mathbb{Q}}$, we have $u=-e_{1}^{*}-\cdots-e_{n}^{*}$. Consider the relation

$$
v+\sum_{i=1}^{n} a_{i} e_{i}=0
$$

and the corresponding class $\gamma \in \mathcal{N}_{1}\left(X_{P}\right)$. We have

$$
-K_{X_{P}} \cdot \gamma=1+\sum_{i=1}^{n} a_{i}=1+\langle v, u\rangle=1+\delta_{P}
$$

Now consider the invariant curve $C_{0} \subset X_{P}$ corresponding to the cone over the face $\operatorname{Conv}\left(e_{2}, \ldots, e_{n}\right)$. There exists $b \in \mathbb{Q}, b \in(0,1]$, such that the numerical class of $C_{0}$ is $b \gamma($ see [19, $\left.\S 2]\right)$. Then $-K_{X_{P}} \cdot C_{0}=b\left(\delta_{P}+1\right) \leq \delta_{P}+1$.

Assume now that $X_{P}$ is smooth, and let $C$ be an invariant curve having minimal anticanonical degree $\iota_{X_{P}}$. The numerical class of $C$ corresponds to a relation $f_{0}+\sum_{i=1}^{n} b_{i} f_{i}=0$, where $b_{i} \in \mathbb{Z}$ and $\operatorname{Conv}\left(f_{1}, \ldots, f_{n}\right)$ is a facet $F$ of $P$ (see [19, §2]). The vertices $f_{1}, \ldots, f_{n}$ are a basis of $N$; if $f_{1}^{*}, \ldots, f_{n}^{*}$ is the dual basis of $M$, then $F=F_{u_{0}}$ with $u_{0}=-f_{1}^{*}-\cdots-f_{n}^{*}$. So

$$
\iota_{X_{P}}=-K_{X_{P}} \cdot C=1+\sum_{i=1}^{n} b_{i}=1+\left\langle f_{0}, u_{0}\right\rangle \geq 1+\delta_{P} \geq \iota_{X_{P}}
$$

and $\iota_{X_{P}}=\delta_{P}+1$.

Proof of Theorem [1. Part (ii) and the inequality in (iii) are straightforward consequences of Theorem 3 and Lemma 2 .

Assume that $\rho_{X_{P}}\left(\iota_{X_{P}}-1\right)=n$. Again using Lemma 2 and Theorem [3, we get $\delta_{P} \geq \iota_{X_{P}}-1>0$ and

$$
|V(P)| \leq n+\frac{n}{\delta_{P}} \leq n+\frac{n}{\iota_{X_{P}}-1}=n+\rho_{X_{P}}=|V(P)|,
$$

so $\iota_{X_{P}}=\delta_{P}+1,|V(P)|=n+\frac{n}{\delta_{P}}$ and the characterization in Theorem 3 (iii) holds. This means that $X_{P}$ is the quotient of $\left(\mathbb{P}^{\delta_{P}}\right)^{\rho_{X_{P}}}$ by a finite subgroup $G$ of the big torus; it is then enough to show that $X_{P}$ is smooth.

We keep the same notation as in the proof of Theorem 3. In order to show the smoothness of $X_{P}$, we have to show that $e_{1}, \ldots, e_{n}$ is a basis of $N$. Let $w \in N$ 
and write $w=\sum_{i=1}^{n} \frac{t_{i}}{s_{i}} e_{i}$ with $t_{i}, s_{i} \in \mathbb{Z}, s_{i} \neq 0$, and $t_{i}, s_{i}$ with no common factors for all $i$.

Suppose that $t_{1} \neq 0$, we show that $s_{1}=1$ (for the other indices the proof is analogous). Consider the relation

$$
f_{\varphi(1)}+\sum_{k \in \varphi^{-1}(\varphi(1))} e_{k}=0
$$

and the corresponding class $\gamma \in \mathcal{N}_{1}\left(X_{P}\right)$. Now consider the invariant curve $C \subset X_{P}$ corresponding to the cone over the face $\operatorname{Conv}\left(e_{2}, \ldots, e_{n}\right)$, and recall that $\operatorname{Conv}\left(e_{1}, \ldots, e_{n}\right)$ and $\operatorname{Conv}\left(f_{\varphi(1)}, e_{2}, \ldots, e_{n}\right)$ are faces of $P$. Then there exists $b \in \mathbb{Q}, b \in(0,1]$, such that the numerical class of $C$ is $b \gamma$ (see [19, §2]). So we have

$$
\iota_{X_{P}} \leq-K_{X_{P}} \cdot C=b\left(-K_{X_{P}} \cdot \gamma\right)=b\left(\delta_{P}+1\right)=b \iota_{X_{P}} \leq \iota_{X_{P}},
$$

which gives $b=1$. This is equivalent to saying that in the quotient lattice $N / N \cap\left(\mathbb{Q} e_{2} \oplus \cdots \oplus \mathbb{Q} e_{n}\right)$, the image $\bar{e}_{1}$ of $e_{1}$ is a generator. Now if $\bar{w}$ is the image

of $w$, we have $s_{1} \bar{w}=t_{1} \bar{e}_{1}$ with $s_{1}, t_{1}$ non zero and with no common factors, so $s_{1}=1$.

Acknowledgments. I am grateful to Benjamin Nill for pointing out to me the relation (41).

\section{References}

[1] Marco Andreatta, Elena Chierici, and Gianluca Occhetta. Generalized Mukai conjecture for special Fano varieties. Central European Journal of Mathematics, 2(2):272-293, 2004.

[2] Victor V. Batyrev. Toric Fano threefolds. Izvestiya Akademii Nauk SSSR Seriya Matematicheskaya, 45(4):704-717, 1981 (in Russian). English translation: Mathematics of the USSR Izvestiya, 19, 13-25, 1982.

[3] Victor V. Batyrev. Dual polyhedra and mirror symmetry for Calabi-Yau hypersurfaces in toric varieties. Journal of Algebraic Geometry, 3:493-535, 1994.

[4] Victor V. Batyrev. On the classification of toric Fano 4-folds. Journal of Mathematical Sciences (New York), 94:1021-1050, 1999.

[5] Laurent Bonavero, Cinzia Casagrande, Olivier Debarre, and Stéphane Druel. Sur une conjecture de Mukai. Commentarii Mathematici Helvetici, 78:601-626, 2003.

[6] Cinzia Casagrande. Toric Fano varieties and birational morphisms. International Mathematics Research Notices, 27:1473-1505, 2003.

[7] Koji Cho, Yoichi Miyaoka, and Nick Shepherd-Barron. Characterizations of projective space and applications to complex symplectic geometry. In Higher Dimensional Birational Geometry, volume 35 of Advanced Studies in Pure Mathematics, pages 1-89. Mathematical Society of Japan, 2002. 
[8] Olivier Debarre. Higher-Dimensional Algebraic Geometry. Universitext. Springer-Verlag, 2001.

[9] Olivier Debarre. Fano varieties. In Higher Dimensional Varieties and Rational Points (Budapest, 2001), volume 12 of Bolyai Society Mathematical Studies, pages 93-132. SpringerVerlag, 2003.

[10] Günter Ewald. Combinatorial Convexity and Algebraic Geometry, volume 168 of Graduate Texts in Mathematics. Springer-Verlag, 1996.

[11] Branko Grünbaum. Convex Polytopes, volume 221 of Graduate Texts in Mathematics. Springer-Verlag, second edition, 2003. First edition 1967.

[12] Benjamin Nill. Complete toric varieties with reductive automorphism group. Preprint math.AG/0407491, 2004.

[13] Benjamin Nill. Gorenstein toric Fano varieties. Manuscripta Mathematica, 116(2):183$210,2005$.

[14] Gianluca Occhetta. A characterization of products of projective spaces. Preprint, available at the author's web page http://www.science.unitn.it/ occhetta/, 2003.

[15] Hiroshi Sato. Toward the classification of higher-dimensional toric Fano varieties. Tôhoku Mathematical Journal, 52:383-413, 2000.

[16] V. E. Voskresenskiǔ and Alexander Klyachko. Toric Fano varieties and systems of roots. Izvestiya Akademii Nauk SSSR Seriya Matematicheskaya, 48(2):237-263, 1984 (in Russian). English translation: Mathematics of the USSR Izvestiya, 24, 221-244, 1985.

[17] Keiichi Watanabe and Masayuki Watanabe. The classification of Fano 3-folds with torus embeddings. Tokyo Journal of Mathematics, 5:37-48, 1982.

[18] Jarosław A. Wiśniewski. On a conjecture of Mukai. Manuscripta Mathematica, 68:135$141,1990$.

[19] Jarosław A. Wiśniewski. Toric Mori theory and Fano manifolds. In Geometry of Toric Varieties, volume 6 of Séminaires et Congrés, pages 249-272. Société Mathématique de France, 2002.

C. Casagrande

Dipartimento di Matematica "L. Tonelli"

UNIVERSITÀ DI PISA

Largo Bruno Pontecorvo, 5

56127 PisA - ITALY

E-mail address: casagrande@dm.unipi.it 